\section{Gelingt der Brusterhalt?}

Die neoadjuvante Chemotherapie ermöglicht auch bei relativ großen Mammakarzinomen häufig eine brusterhaltende Operation. Bei einigen Frauen ist sie aber nicht erfolgreich. Um ihnen eine unnötige Belastung ohne Erfolgsaussichten zu ersparen, wäre das verlässliche Identifizieren dieser Subgruppe wünschenswert.

—ür Östrogenrezeptor(ER)-positive F und nodal negative Mammakarzinome wurde ein Brustkrebsindex (Breast Cancer Index, BCI) entwickelt, um das Rezidivrisiko abzuschätzen. Er beruht auf der Kombination verschiedener Gentests: zum einen des Verhältnisses von HOXB13 zu IL17BR, zum anderen eines Index, der anhand von fünf verschiedenen Genen ermittelt wird. Der BCI führt zu einer Einteilung in drei Risikokategorien hinsichtlich der Fernmetastasierung. Jetzt wurde er auch zur Abschätzung der Chemosensitivität ER-positiver und nodal negativer Mammakarzinome vor Beginn einer neoadjuvanten Chemotherapie eingesetzt. Dazu wurde Tumorgewebe von 150 Mammakarzinom-Patientinnen, die sich einer neoadjuvanten Therapie unterzogen, mit Real-TimePCR untersucht. Gemäß des BCI fielen
$42 \%$ der Patientinnen in die Kategorie des geringsten Risikos, $35 \%$ in die mit mittlerem und $23 \%$ in die mit hohem Risiko. Die BCI-Risikokategorien waren signifikant mit dem Tumorgrad und dem ER/Progesteronrezeptor(PR)-Status assoziiert.

Der negative prädiktive Wert betrug bei geringem Risiko nach BCI $98,4 \%$ bezogen auf das pathologisch komplette Ansprechen (pCR) und 86\% bezüglich des Brusterhalts. Bei Frauen der höchsten BCI-Risikokategorie war unabhängig von klinisch-pathologischen Risikofaktoren wie Alter, ER/PR-Verhältnis, Tumorgrad, Tumorgröße und HER2Status ein pCR 34-mal und der erfolgreiche Brusterhalt 5,8-mal wahrscheinlicher als bei Frauen der niedrigen Risikokategorie ( $\mathrm{p}=0,0055$ bzw. $\mathrm{p}=0,0022$ ). Bei über $98 \%$ der Frauen mit den nied- rigsten BCI-Werten sprechen die Karzinome nicht optimal auf die neoadjuvante Therapie an und sie profitieren höchstens gering von dieser Behandlung. In der Gruppe mit den höchsten Werten sind es dagegen nur $29 \%$. Bei einem niedrigen BCI-Wert ist also möglicherweise eine neoadjuvante Therapie wenig erfolgversprechend. Dies muss aber in weiteren Studien erst noch eingehender untersucht werden.

Fazit: Die mithilfe des BCI identifizierten Risikokategorien können zusätzlich $\mathrm{zu}$ anderen klinisch-pathologischen Faktoren helfen, die Erfolgschancen einer neoadjuvanten Therapie bezüglich Chemosensitivität des Tumors (pCR) und Brusterhalt abzuschätzen sowie $\mathrm{Pa}$ tientinnen zu identifizieren, die von der neoadjuvanten Therapie nicht profitieren. Die hohen negativen Prädiktionswerte sprechen dafür, dass dieser Test tatsächlich klinisch relevant werden könnte.

Friederike Klein

Mathieu MC et al. Breast Cancer Index predicts pathological complete response and eligibility for breast conserving surgery in breast cancer patients treated with neoadjuvant chemotherapy. Ann Oncol. 2012;23(8):2046-52.

\title{
Sechs Zyklen nicht besser als vier
}

\section{Welches die ideale Dauer der adjuvanten Chemotherapie bei Frauen mit einem Niedrig-Risiko-Mammakarzinom ist, wurde in einer aktuellen Studie untersucht.}

$\mathrm{n}$ der randomisierten Phase-III-Studie 40101 der Cancer and Leukemia Group B (CALGB 40101) gingen Lawrence N. Shulman und Kollegen bei 3.171 Frauen mit operablem Mammakarzinom - 6\% mit 1-3 positiven Lymphknoten - der Frage nach, ob sechs Chemotherapiezyklen besser sind als vier. Die Chemotherapie bestand entweder aus Paclitaxel ( 80 $\mathrm{mg} / \mathrm{m}^{2}$ einmal wöchentlich oder $175 \mathrm{mg} /$ $\mathrm{m}^{2}$ alle zwei Wochen) oder aus Doxorubicin $\left(60 \mathrm{mg} / \mathrm{m}^{2}\right)$ und Cyclophosphamid $\left(600 \mathrm{mg} / \mathrm{m}^{2}, \mathrm{AC}\right)$ alle drei oder alle zwei Wochen.

Primärer Endpunkt war das rezidivfreie Überleben. Nach median 5,3 Jahren blieb sowohl das rezidivfreie als auch das Gesamtüberleben von der Therapiedau- er unbeeinflusst. Das rezidivfreie 4-Jahres-Überleben betrug $90,9 \%$ in der Gruppe mit sechs Zyklen und $91,8 \%$ in der Gruppe mit vier Zyklen (adjustierte Hazard Ratio[HR] 1,03; p = 0,77); beim 4-Jahres-Gesamtüberleben lagen die Werte bei 95,3 bzw. 96,3\% (HR 1,12; $\mathrm{p}=$ 0,44). In zusätzlichen Analysen konnte keine Subgruppe identifiziert werden, die von der längeren Therapiedauer profitiert hätte. Auch hinsichtlich des verwendeten Therapieregimes gab es keine Unterschiede.

Erwartungsgemäß traten Toxizitäten unter AC häufiger auf als unter Paclitaxel, zudem waren die Toxizitäten unter sechs Zyklen AC etwas häufiger als unter vier Zyklen.
Bei der Interpretation der Ergebnisse ist zu berücksichtigen, dass die beiden Therapieregime kombiniert ausgewertet wurden und dass Paclitaxel allein bisher nicht als Standardtherapie anzusehen ist. Außerdem ist zu beachten, dass über $90 \%$ der Studienteilnehmerinnen keine befallenen Lymphknoten hatten und $77 \%$ HER2-negativ waren.

Fazit: Eine Verlängerung der Chemotherapie von vier auf sechs Zyklen kann Frauen mit reseziertem primärem Mammakarzinom und maximal drei positiven Lymphknoten erspart werden. Sie brachte in der vorliegenden Studie keinen zusätzlichen Nutzen. Judith Neumaier

Shulman LN et al. Six cycles of doxorubicin and cyclophosphamide or paclitaxel are not superior to four cycles as adjuvant chemotherapy for breast cancer in women with zero to three positive axillary nodes: Cancer and Leukemia Group B 40101. J Clin Oncol. 2012; 23. Juli 2012; DOI: 10.1200/JCO.2011.40.6405. 